\title{
Speed Control of DC Motor Using PSO Tuned PI Controller
}

\author{
Atulit Patel ${ }^{1}$ Kapil Parikh ${ }^{2}$
}

\begin{abstract}
The control of DC motor system is difficult and mathematically tedious due to their high non linearity property. To overcome this difficulty, a new approach has been required. This paper presented a systematic procedure to develop PI based speed controller for DC motor. The designing of speed controller through state estimation approaches. The optimal parameter of PI controller is finding out by using PSO algorithms.The performances and characteristics of DC motor are observed. The simulation results verify that steady state error is reduced, the rising time is improved and the disturbances affect is reduced hence the better performances of DC motor.
\end{abstract}

Keywords: -DC motor, Linear, Particle Swarm Optimization, State Estimation, Speed Controller

\section{Introduction}

The DC motors are in general much more adaptable speed drives than AC motors which are associated with a constant speed rotating field. It is observed that most of the industry is operating under stress condition further load parameter and control variable exhibit uncertainness in real practice and in fact these are random variables. Calculated values of load variable normally contain various inaccuracies.

It has been observed that error may vary in the range of 5-10\%. A few percentage error may be required tolerable in the area of the load speed controlling where these inaccuracies in the entire controller. In such situation minor inaccuracy in speed control are of little concern. Further the speed controller can always be designed to have sufficiently low effect on the non linearity of DC motor; so as to worst effect of parameter uncertainty can be accounted. In real time operation, the situation is different; design controller may encounter situation never imagined by designer before it took its present shape. Hence, in real time operation condition, risk of affecting nonlinearity of motor is always present. Here it is designed a controller which not affects the nonlinearity in DC motor.

\section{DcMotor}

The stator of the DC motor has poles, which are excited by DC current to produce Magnetic fields. The rotor has a ring-shaped laminated iron-core with slots. Coils with several turns are placed in the slots. The distance between the two legs of the coil is about 180 electric degrees. DC motors are characterized by their versatility. By means of various combinations of shunt, series and separately excited field winding they can be designed to display a wide variety of volt ampere or speed torque characteristics for both dynamic and steady state operation. The separately excited dc motor model is chosen for its good electrical and mechanical performances rather than other DC motor models. The DC motor is driven by applied voltage. In DC motor, the torque may be controlled by varying the armature current or field current. One of these is varied to control the torque while the other is held constant.

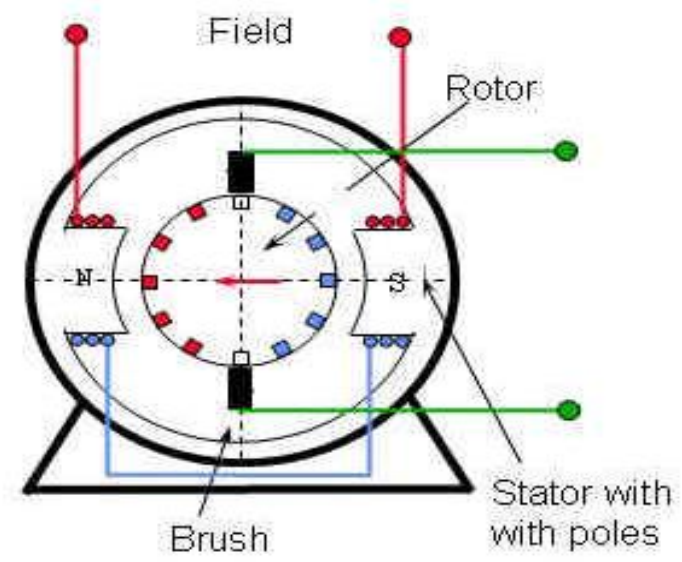

Fig. 1: Basic diagram of DC motor 
Dynamic behavior of DC motor followed by:

$$
\begin{aligned}
& \frac{d i_{a}}{d t}=\mathrm{V} / \mathrm{L}_{\mathrm{a}}-\mathrm{K}_{\mathrm{b}} \omega / \mathrm{L}_{\mathrm{a}}-\mathrm{R}_{\mathrm{a}} \mathrm{i}_{\mathrm{a}} / \mathrm{L}_{\mathrm{a}} \\
& \frac{\mathrm{d} \omega}{\mathrm{dt}}=-\mathrm{B}_{1} \omega / \mathrm{J}+\mathrm{K}_{\mathrm{m}} \mathrm{i}_{\mathrm{a}} / \mathrm{J}-\mathrm{T}_{\mathrm{L}} / \mathrm{J}
\end{aligned}
$$

State-space equation for dc motor:

$\frac{\mathrm{dx}}{\mathrm{dt}}=\mathrm{Ax}+\mathrm{Bu}, \quad \mathrm{y}=\mathrm{Cx}+\mathrm{Du}$

$A=\left[\begin{array}{cc}-\frac{R_{a}}{L_{a}} & \frac{-K_{b}}{L_{a}} \\ \frac{K_{m}}{J} & \frac{-B_{1}}{J}\end{array}\right], \quad B=\left[\begin{array}{c}\frac{1}{L_{a}} \\ 0\end{array}\right]$

$\mathrm{C}=\left[\begin{array}{ll}0 & 1\end{array}\right], \quad \mathrm{D}=\left[\begin{array}{ll}0 & 0\end{array}\right]$

\section{State Estimation}

A state feedback control law $\mathrm{u}=-\mathrm{Kx}$ utilized for finding the complete system state $\mathrm{x}$, When the complete state is not available for feedback. If the system is observable, then we can design a state estimator, an artificial dynamical system whose state converges to the actual system state. Consider the LTI state equation $\dot{x}=A x+B u$

If A is Hurwitz (i.e., its eigenvalues lies in the open left half plane). Assuming that these equations describe the system dynamics, define the fictitious system

$\dot{\tilde{x}}=A \tilde{x}+B u$

Where $\mathrm{x}$ represents the state estimate. The state estimate is therefore available for use by the controller. Even if the initial state of the estimator disagrees with the actual initial state, the state estimate will converge to the true state because the state matrix is Hurwitz. To see this, define the error coordinate are -

$e=x-\tilde{x}$

\section{Observer Design}

The model of a DC motor is given by:

$\left[\begin{array}{c}\dot{I_{a}} \\ \dot{\omega}\end{array}\right]=\left[\begin{array}{cc}-\frac{R_{a}}{L_{a}} & \frac{-K_{b}}{L_{a}} \\ \frac{K_{m}}{J} & \frac{-B_{1}}{J}\end{array}\right]\left[\begin{array}{c}I_{a} \\ \omega\end{array}\right]+\left[\begin{array}{c}\frac{1}{L_{a}} \\ 0\end{array}\right] V+\left[\begin{array}{c}0 \\ \frac{-1}{J}\end{array}\right] T_{L}$

Where $\omega, i_{a}$, and $V$ are rotor speed, armature current and armature voltage respectively, $T_{L}$ is load torque, $R_{a}$ and $L_{a}$ are armature resistance and inductance, $\mathrm{Km}$ is torque constant, which is equal to back emf constant $\mathrm{K}_{\mathrm{b}}$, and $B_{l}$ and $J$ are dynamic friction constant and inertia respectively. Based on the fact that the states of two DC servo motors with identical parameters converge to the same trajectories under the same input voltage and load torque regardless of their initial conditions, an observer which is exactly in the same form as the actual motor model can be designed without using any feedback as

$\left[\begin{array}{c}\dot{\tilde{I}} \\ \dot{\tilde{\omega}}\end{array}\right]=\left[\begin{array}{cc}-\frac{R_{a}}{L_{a}} & \frac{-K_{b}}{L_{a}} \\ \frac{K_{m}}{J} & \frac{-B_{1}}{J}\end{array}\right]\left[\begin{array}{c}\widetilde{I_{a}} \\ \widetilde{\omega}\end{array}\right]+\left[\begin{array}{c}\frac{1}{L_{a}} \\ 0\end{array}\right] V+\left[\begin{array}{c}0 \\ \frac{-1}{J}\end{array}\right] T_{L}$

Where the observer variables and parameters are shown with the addition of tilde $(\sim)$ to their symbols in the actual system. Such a system is called anobserver.

\section{Full Order Observer}

A state observer estimates the state variables based on the measurement of the output and control variables. State observer can be designed if and only if the observability condition is satisfied which is - 
$W_{o}=\left[\begin{array}{c}C \\ --- \\ C A \\ --- \\ \cdot \\ \cdot \\ --- \\ C A^{n-1}\end{array}\right]$

Assume that the state $\mathrm{X}$ is to be approximated by the state $\dot{\tilde{x}}$ of the dynamic model $\dot{\tilde{x}}=\left(A-K_{e} C\right) \tilde{x}+B u+K_{e} y$

Which represent the state observer the state observer has y and u as input and $\dot{\tilde{x}}$ as output and $\mathrm{K}_{\mathrm{e}}$ is the observer gain

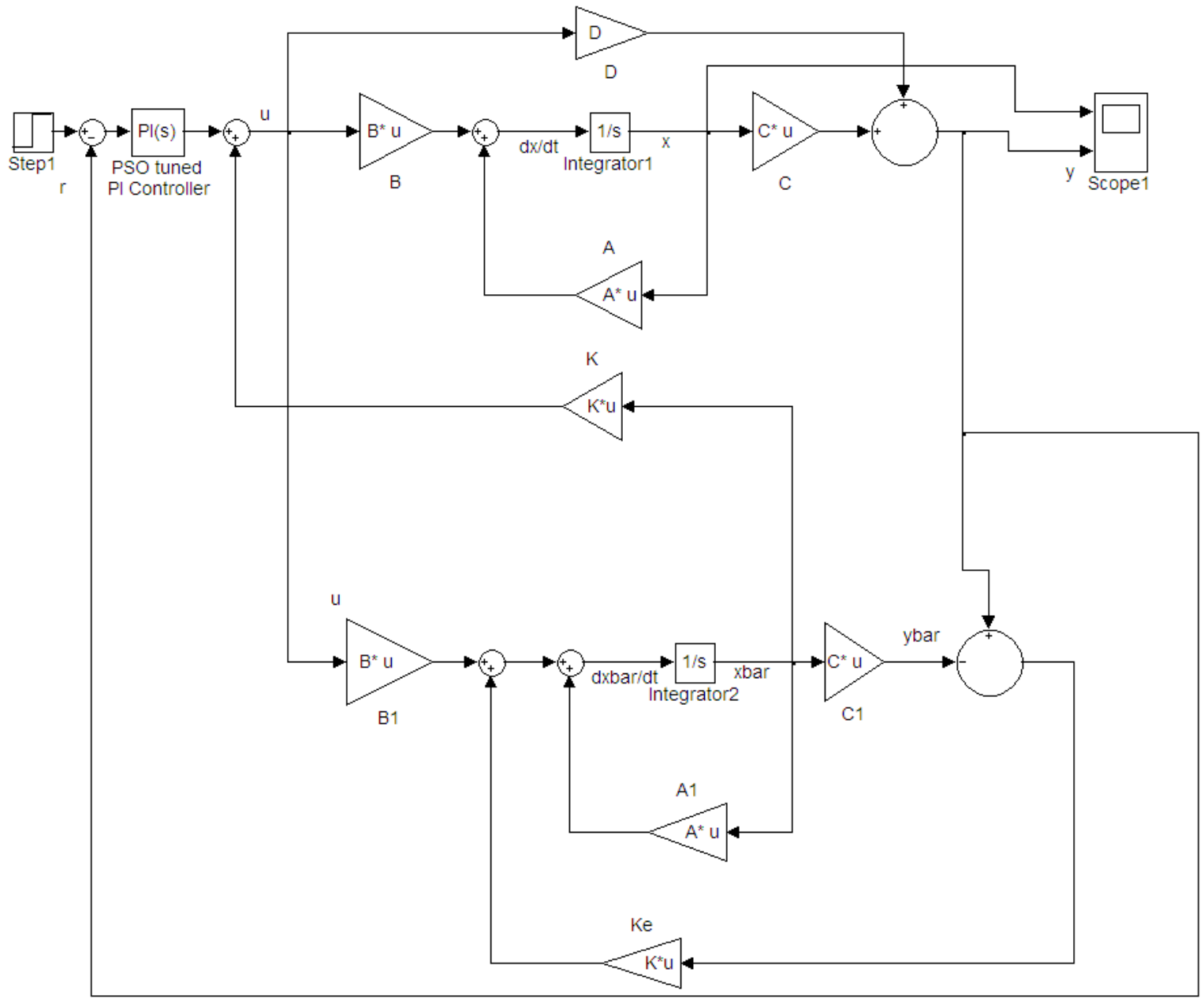

Fig. 2: Matlab model of observer

\section{PI Controller}

PID tuning is a complex problem, even though there are only three parameters and in principle is easy to evaluate, because it must satisfy complex criteria within the limitations of PID control. PI control with its two term functionality covering treatment to both transient and steady state response, offers the simplest and yet most efficient solution to many real world control problems. In spite of the simple structure and robustness of this controller, optimally tuning gains of PI controllers have been quite difficult. When the control problem is to regulate the process output around a set point, it is natural to consider error as an input, and it follows that the integral of the error. 


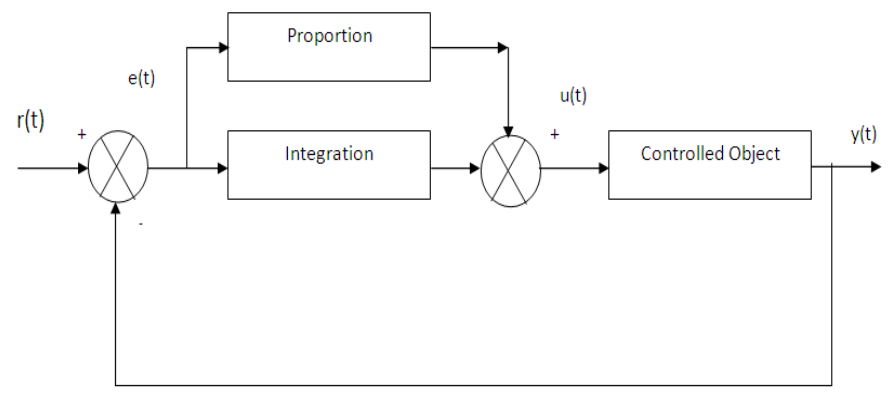

Fig. 3: PI Controller

\section{Particle Swarm Optimization}

PSO is a population-based optimization method first proposed by Eberhart and Colleagues. Some of the attractive features of PSO include the ease of implementation and the fact that no gradient information is required. It can be used to solve a wide array of different optimization problems. Like evolutionary algorithms, PSO technique conducts search using a population of particles, corresponding to individuals. Each particle represents a candidate solution to the problem at hand. In a PSO system, particles change their positions by flying around in a multidimensional search space until computational limitations are exceeded. Concept of modification of a searching point by PSO is shown in Fig. 4.

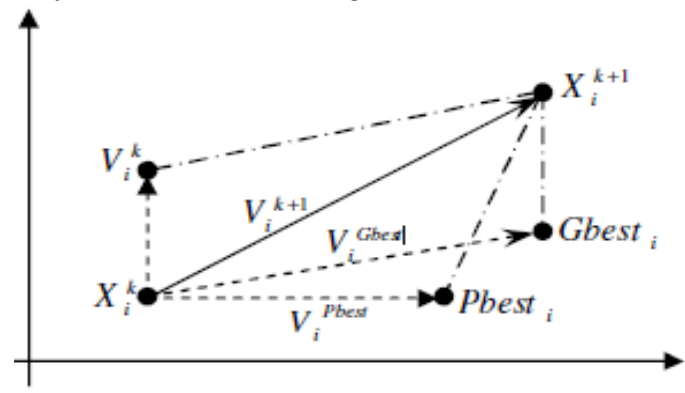

Where-

$\mathrm{X}_{\mathrm{k}} \quad$ : Current Position,

$\mathrm{X}_{\mathrm{k}+1}$ : Modified position

$\mathrm{V}_{\mathrm{k}} \quad$ : Current Velocity
Fig. 4: Parameter Searching by PSO algorithm.

$$
\begin{aligned}
& \mathrm{V}_{\mathrm{k}+1}: \text { Modified velocity } \\
& \mathrm{V}_{\text {Pbest }}: \text { Velocity based on Pbest } \\
& \mathrm{V}_{\text {Gbest }} \text { : Velocity based on Gbest }
\end{aligned}
$$

VIII. Simulation \& Results

The DC motor model as defined in equations above is simulated by using MATLAB. Results show the good performance of the proposed PSO tuning method. The PI controller is introduced in system and performance is observed.

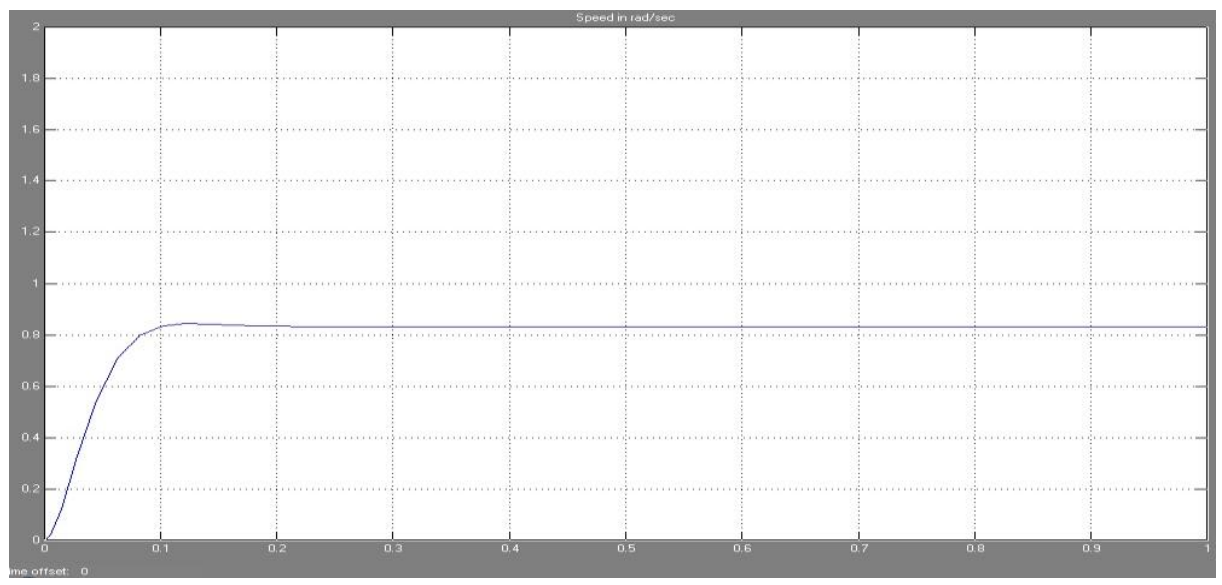

Fig. 5: Speed waveform of dc motor without observer 


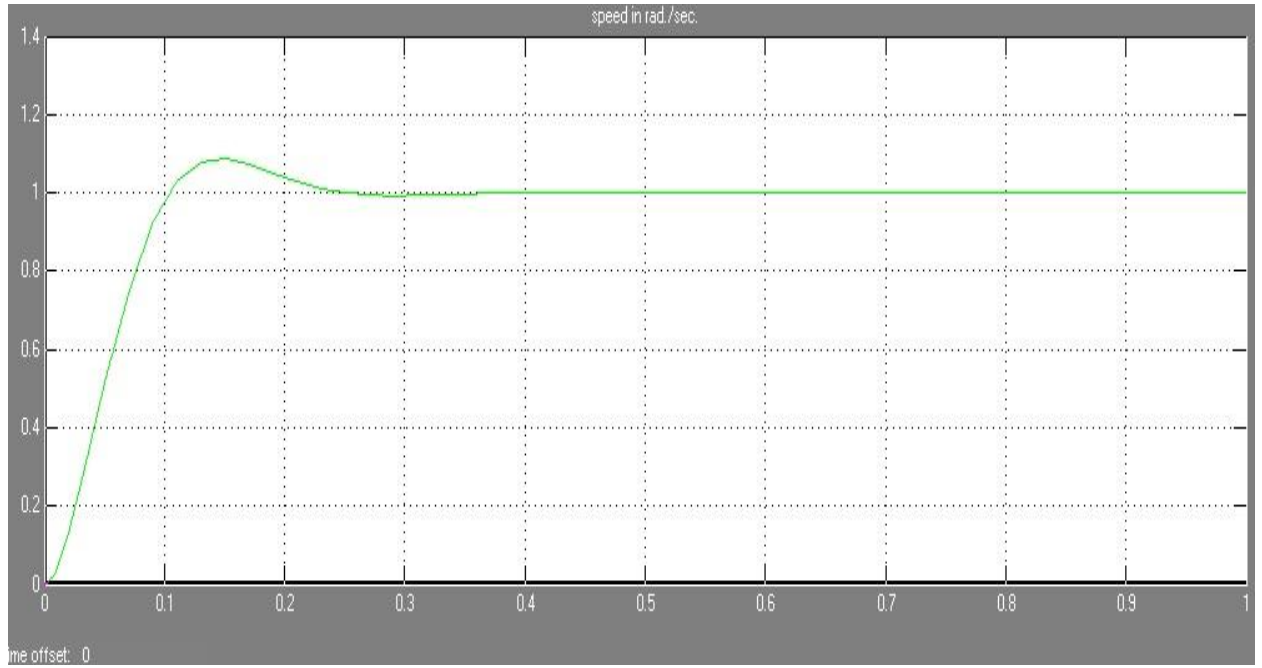

Fig. 6: Speed waveform of dc motor with observer

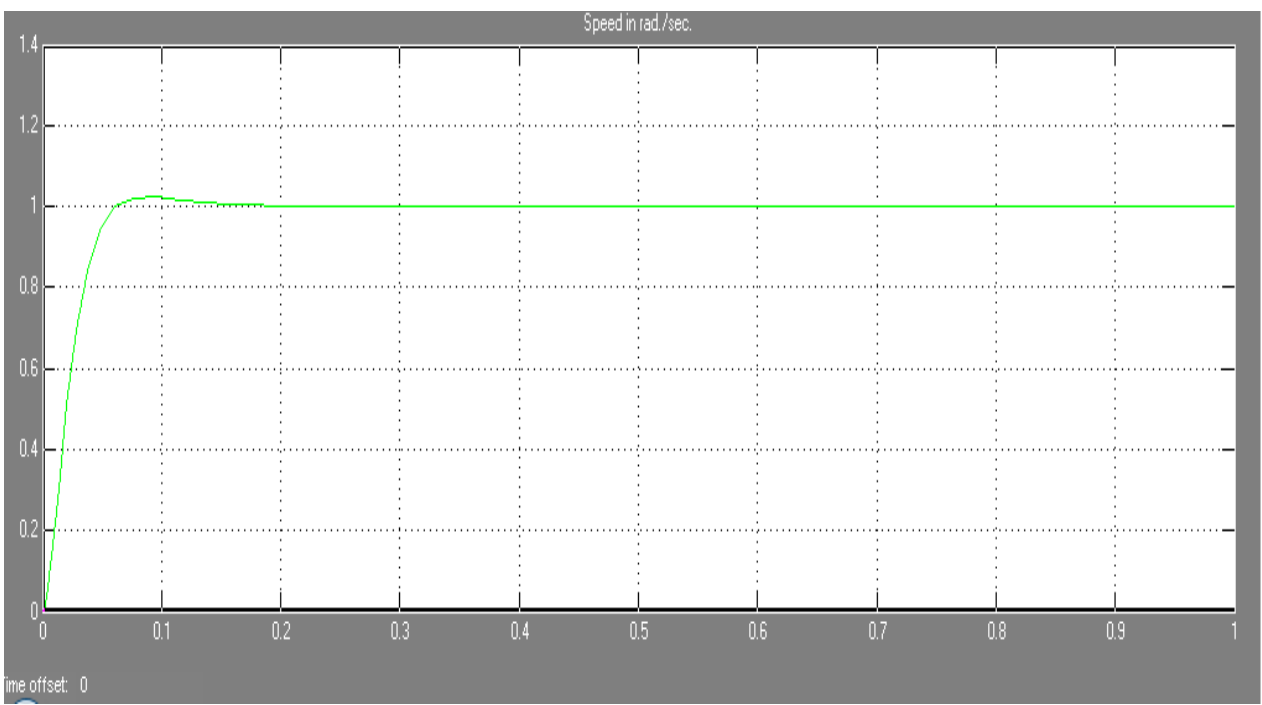

Fig. 7: Speed waveform of state feedback controller with PSO tuned PI controller

\section{Conclusion}

The behavior of DC motor with PSO tuned PI controller is studied. PSO algorithm is used for optimum tuning of PI controller. This model presented to find out optimal parameter of PI controller using the PSO. The results show that the proposed PI controller can perform an efficient performance for speed controlling of DC motor. This model can improve the dynamic performance of the system in a better way. The PI-PSO controller is the best which presented satisfactory performances and possesses good robustness (no overshoot, minimal rise $\&$ peak time, Steady state error $=0$ ).

\section{References}

[1]. C. M. Lim "Experimental study of a fuzzy system for dc motor control", Proceedings of Asia-Pacific Workshop on Advances in Motion Control, 1993

[2]. P. B. Schmidt and R. D. Lorenz "Design principles and implementation of acceleration feedback to improve performance of dc drives", IEEE Trans. Industrial Applications, vol. 28, pp.594 -599, 1992.

[3]. W. Leonhard, Control of Electrical Drives 1985.

[4]. M. Iwasaki , K. Seki and Y. Maeda "High-precision motion control techniques: A promising approach to improving motion performance", IEEE Ind. Electron. Mag., vol. 6, no. 1, pp.32 -40 2012.

[5]. Y. Dong, J. Tang, B. Xu and D. Wang," An Application of swarm optimization to Non-Linear programming", Computers and Mathematics with applications 49 (2005), ELSVIER LTD, pp. 1655-1668, 2005.

[6]. J. C. Basilio and S. R. Matos, "Design of PI and PID Controllers With Transient Performance Specification", IEEE Trans. Education, vol. 45, Issue No. 4, pp. 364-370, 2002 